\title{
STRONG GRAVITATIONAL SLINGSHOT EFFECT IN CENTRA PARTS OF STELLAR AND GALACTIC SYSTEMS
}

\author{
J. ANOSOVA \\ ASE, University of Texas at Austin, Austin TX 78712, USA
}

We study numerically the dynamical evolution of models of stellar systems which contain two heavy centers of gravity and extended shells inside and around orbits of these binaries. Initially the binary components surrounded by numerous small-mass particles on shells with small initial velocities. It is shown that at some moment of time there is a collapse of these particles on to the heavier component of the binary. We have the strong interactions of bodies at this time. Frequently, the 'gravitational slingshot' effect occurs. Further in time, some part of particles which initially were outside the binary orbit escape from the system. Other particles are captured by binary components forming in the center a 'dumb-bell' bar. During evolution of our models different kinds of structures of systems often are very similar to the observed structures of galaxies: spiral and elliptical galaxies, interacting galaxies, different kinds of flows and jets. Totally systems are expanding. Formation of different kinds of structures of galaxies depends on motions of particles with respect to the line of apses of the nuclear binary: 1 . in the case of almost orthogonal motion (the gravitational slingshot effect), these particles escape from systems and before an escape form open expanding spirals; 2 . in the alternative case (small angles of motion of the particles with respect to this line), these particles are captured by the binary components; 3 . in the intermediate cases, particles form different kinds of flows like jets, rings, close spirals etc.

Fig.1 shows basic dynamical mechanisms for the formation of various nuclear jets and flows caused by strong interactions with a binary nucleus. This figure presents the trajectories of small mass bodies during strong interactions with the center of mass of a system. The filled circles show the trajectories of escaping particles; the open squares show the motions of particles that return to the center of inertia of a system after a failed ejection. Other symbols show trajectories of particles that form different 
types of large and small rings inside a system. A part of the large circle in the negative quadrant $(x<0, y<0)$ shows the initial positions of the small-mass particles on a shell around a massive binary. The filled squares show schematically the boundaries of regions of initial positions of particles on a shell with different kinds of final motions.

\section{References}

Agekian,T., Anosova J., (1967) Sov.Astron.J.,44, 1267.

Agekian,T., Anosova J., (1991) Sov.Astron.J.,68, 1099.

Anosova J., (1986) Astrophys. Space Sci.,124, 217.

Anosova J., (1991) Celest.Mech.\&Dynam.Astron.,51, 1.

Toomre,A.,Toomre,J., (1972) Astrophys.J.,178, 623.

Valtonen,M., (1988) Vistas Astron.,32, 23.

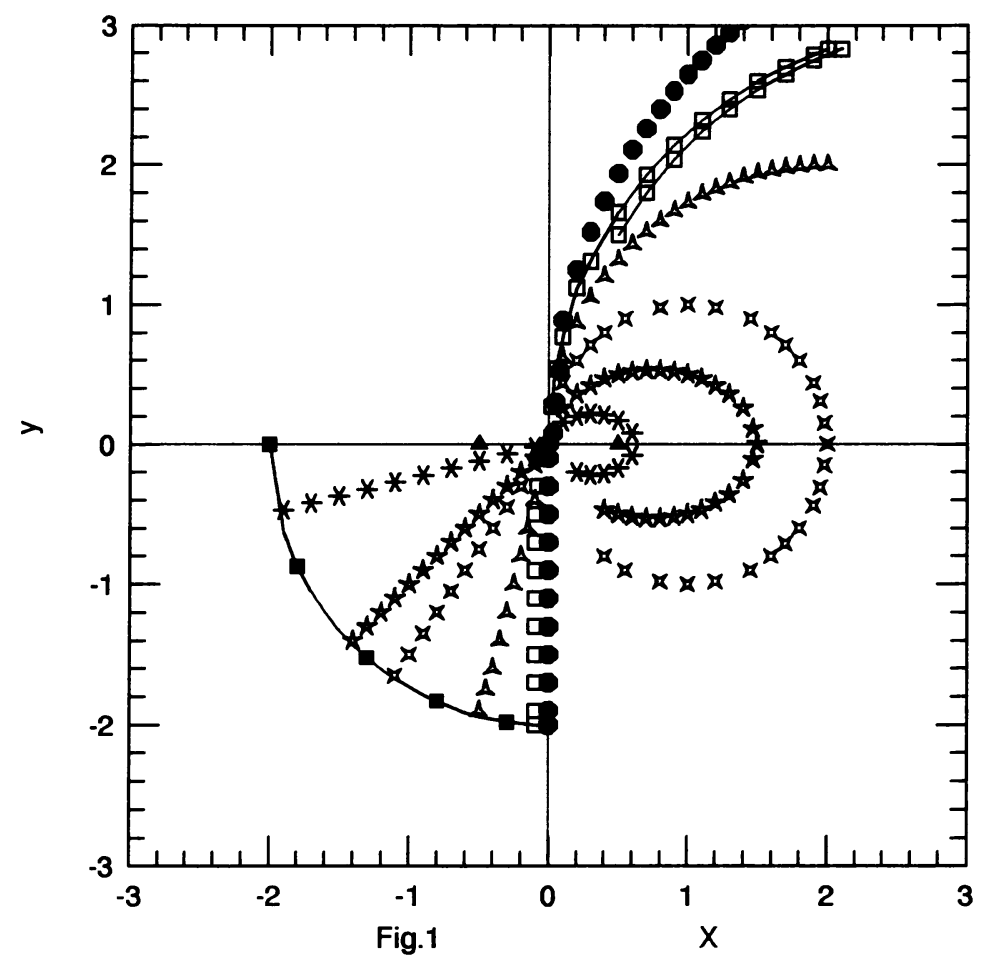

